\title{
Review of: "Diabetes Mellitus and COVID-19: Review Article"
}

Mohammad Chand Jamali

Potential competing interests: The author(s) declared that no potential competing interests exist.

Well written analysis. 